\title{
ARTICLE
}

\section{Measurement of neutron energy spectra behind shields for quasi-monoenergetic neutrons generated by 246-MeV and 389-MeV protons using a Bonner sphere spectrometer}

\author{
Tetsuro Matsumoto ${ }^{\mathrm{a}^{*}}$, Akihiko Masuda ${ }^{\mathrm{a}}$, Jun Nishiyama ${ }^{\mathrm{b}}$, Hideki Harano ${ }^{\mathrm{a}}$, Hiroshi Iwase ${ }^{\mathrm{c}}$, Yosuke Iwamoto ${ }^{\mathrm{d}}$, Masayuki \\ Hagiwara $^{\mathrm{c}}$, Daiki Satoh ${ }^{\mathrm{d}}$, Hiroshi Yashima ${ }^{\mathrm{e}}$, Yoshihiro Nakane ${ }^{\mathrm{d}}$, Hiroshi Nakashima ${ }^{\mathrm{d}}$, Yukio Sakamoto ${ }^{\mathrm{d}}$, Christian \\ Pioch $^{\mathrm{f}}$, Vladimir Mares ${ }^{\mathrm{f}}$, Atsushi Tamii ${ }^{\mathrm{g}}$, Kichiji Hatanaka ${ }^{\mathrm{g}}$ and Takashi Nakamura ${ }^{\mathrm{h}}$ \\ ${ }^{a}$ National Institute of Advanced Industrial Science and Technology, 1-1-1 Umezono, Tsukuba, Ibaraki, 305-8568 Japan; ${ }^{b}$ Tokyo \\ Institute of Technology, 2-12-1 O-okayama, Meguro-ku, Tokyo, 152-8350 Japan; ${ }^{c}$ High Energy Accelerator Research \\ Organization (KEK), 1-1 Oho, Tsukuba, Ibaraki, 305-0801 Japan; ${ }^{d} J a p a n$ Atomic Energy Agency, 2-4 Shirakata, Tokai-mura, \\ Naka-gun, Ibaraki-ken, 319-1195, Japan; ${ }^{e}$ Kyoto University, 2-1010 Asayo-nishi, Kumatori-cho, Sennan-gun, Osaka, 590-0494 \\ Japan; ${ }^{f}$ German Reserach Center for Enviromental Health, 85764 Neuherberg, Germany; ${ }^{g}$ Research Center for Nuclear Physics, \\ Osaka University, 10-1 Mihogaoka, Ibaraki, Osaka, 567-0047 Japan; ${ }^{h}$ Tohoku University, 6-3 Aoba, Aramaki, Aoba-ku, Sendai, \\ Miyagi, 980-8578 Japan
}

\begin{abstract}
Neutron energy spectra behind concrete and iron shields were measured for quasi-monoenergetic neutrons above $200 \mathrm{MeV}$. Quasi-monoenergetic neutrons were produced by the ${ }^{7} \mathrm{Li}(\mathrm{p}, \mathrm{xn})$ reaction with $246-\mathrm{MeV}$ and $389-\mathrm{MeV}$ protons. A Bonner sphere spectrometer was used to obtain the neutron energy spectra. Shielding materials are concrete blocks with thicknesses from $25 \mathrm{~cm}$ to $300 \mathrm{~cm}$ and iron blocks with thicknesses from $10 \mathrm{~cm}$ to $100 \mathrm{~cm}$. The neutron energy spectra behind the concrete and iron shields were obtained by the unfolding method using the MAXED code. Ambient dose equivalents were obtained as a function of a shield thickness successfully. These experimental data will be useful to perform accurate shielding design, benchmark calculation codes and evaluate neutron cross section data.
\end{abstract}

Keywords: quasi-monoenergetic neutrons; Bonner sphere spectrometer; shielding experiments; concrete; iron; unfolding; dose distribution

\section{Introduction}

Recently, high-energy and intense beam accelerators, such as the Japan Proton Accelerator Research Complex (J-PARC) are used for various studies and industries. In these facilities, secondary neutrons with energies above $100 \mathrm{MeV}$ are produced around accelerators and beam lines by nucleon-nucleus and nucleus-nucleus reactions. For accurate shielding design of high energy accelerators, experiment data are indispensable to construction of these facilities from the point of view of radiation protection. High-energy neutron penetration data for main shielding materials (concrete and iron) are very important. However, the shielding experimental data for high energy neutrons above $100 \mathrm{MeV}$ are still insufficient both in quality and in quantity as compared with those below $100 \mathrm{MeV}$ [1-5].

In this study, we measured the neutron energy spectra behind concrete and iron shields using a Bonner sphere spectrometer (BSS) for quasi-monoenergetic neutrons produced by the ${ }^{7} \mathrm{Li}(\mathrm{p}, \mathrm{xn})$ reaction with $246-\mathrm{MeV}$ and $389-\mathrm{MeV}$ protons at the Research Center for Nuclear Physics (RCNP) of the Osaka University. The experimental data are used to investigate accuracy of calculation codes and evaluate neutron cross section data.

\section{Experiments}

\subsection{Neutron source}

Figure 1 shows a schematic view of a typical experimental arrangement. Shielding experiments were performed at the time-of-flight (TOF) beam course of RCNP. Quasi-monoenergetic neutrons with peak energies of $243.5 \mathrm{MeV}$ and $386.6 \mathrm{MeV}$ were produced by the ${ }^{7} \mathrm{Li}(\mathrm{p}, \mathrm{xn})$ reaction by bombarding a $1-\mathrm{cm}$ thick $\mathrm{Li}$ target with $246-\mathrm{MeV}$ and $389-\mathrm{MeV}$ protons from the AVF cyclotron and the ring cyclotron of RCNP. A neutron beam emitted in the forward direction was

\footnotetext{
*Corresponding author. Email: t-matsumoto@aist.go.jp
} 


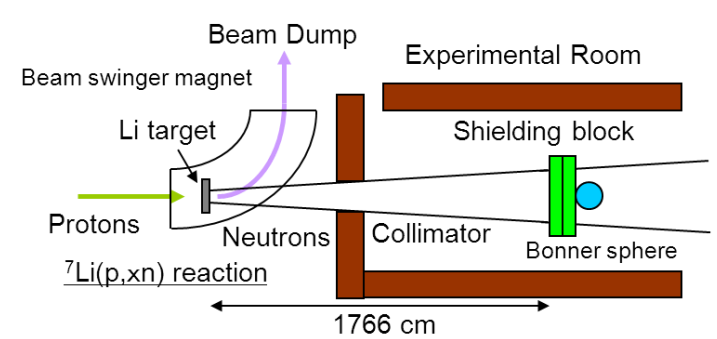

Figure 1. Schematic view of a typical experimental arrangement at RCNP.

extracted into the TOF room through an iron collimator of $12-\mathrm{cm}$ wide and $10-\mathrm{cm}$ high aperture embedded inside a concrete wall with a thickness of $15 \mathrm{~cm}$. The proton beam penetrated through the target was guided to a beam dump using a beam swinger magnet. Neutron source spectra in the TOF room were measured with liquid scintillators by means of the TOF method [6].

\subsection{Setup}

Shielding materials are concrete blocks $\left(2.33 \mathrm{~g} / \mathrm{cm}^{3}\right)$ with thicknesses from $25 \mathrm{~cm}$ to $300 \mathrm{~cm}$ and iron blocks $\left(7.87 \mathrm{~g} / \mathrm{cm}^{3}\right)$ with thicknesses from $10 \mathrm{~cm}$ to $100 \mathrm{~cm}$. The components of concrete are given in Table 1. The distance between the Li target and upstream surface of the shielding materials was $17.7 \mathrm{~m}$. The BSS was contact with the backward surface of shielding material.

\subsection{Bonner sphere spectrometer}

Neutron energy spectra behind the shields were measured with the BSS [7]. The BSS consists of a ${ }^{3} \mathrm{He}$ spherical proportional counter (CENTRONIC LTD, SP9, gas pressure: $21.3 \mathrm{kPa}$ ) and polyethylene (PE, 0.95 $\mathrm{g} / \mathrm{cm}^{3}$ ) moderators with diameters from $7.62 \mathrm{~cm}$ to 24.1 $\mathrm{cm}$. In addition, measurements were performed using inserting metal shells made of lead (457p: 10.2-cm-diameter PE sphere $+1.27-\mathrm{cm}$ thick $\mathrm{Pb}$ shell + 2.54-cm thick PE shell) and copper (457c: 10.2-cm-diameter PE sphere + 1.27-cm-thick $\mathrm{Cu}$ shell + 2.54-cm thick PE shell) [8]. The response functions of BSS were simulated with the MCNPX code [9]. The JENDL-HE [10] file was used in the simulation. The responses at neutron energies of $144 \mathrm{keV}, 565 \mathrm{keV}, 5.0$ $\mathrm{MeV}$ and $14.8 \mathrm{MeV}$ were calibrated in the mono-energetic neutron standard fields [11] of the National Institute of Advanced Industrial Science and Technology. The calibrated and the calculated results are in good agreement within measurement uncertainties. Responses for 243.5-MeV and 386.6-MeV mono-energetic neutrons were also experimentally evaluated by the two-angle differential measurements, which was described in detail elsewhere [7]. Figure 2 shows the response function of the BSS.

However, because the BSS was contacted with the surface of shielding block, actual response functions were affected by the neutron multi-scattering effect between the moderator of BSS and the shielding block, called ping-pong effect. The response function was also
Table 1. Components of concrete

\begin{tabular}{|c|c|c|c|}
\hline Nuclide & $\begin{array}{c}\text { Atomic density } \\
\left(10^{22} \mathrm{~cm}^{-3}\right)\end{array}$ & Nuclide & $\begin{array}{c}\text { Atomic density } \\
\left(10^{22} \mathrm{~cm}^{-3}\right)\end{array}$ \\
\hline $\mathrm{H}$ & 1.47 & $\mathrm{Si}$ & 0.419 \\
\hline $\mathrm{C}$ & 0.836 & $\mathrm{~S}$ & 0.018 \\
\hline $\mathrm{O}$ & 4.13 & $\mathrm{~K}$ & 0.00375 \\
\hline $\mathrm{Na}$ & 0.0194 & $\mathrm{Ca}$ & 0.987 \\
\hline $\mathrm{Mg}$ & 0.0297 & $\mathrm{Fe}$ & 0.0192 \\
\hline $\mathrm{Al}$ & 0.0725 & & \\
\hline
\end{tabular}

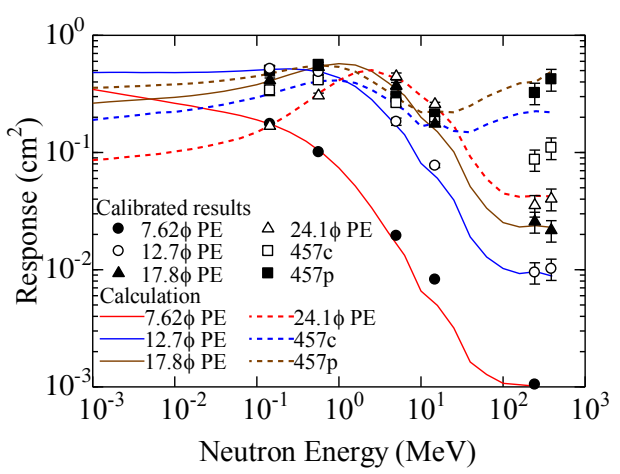

Figure 2. Calculated response function of the BSS. The calibrated results are also shown.

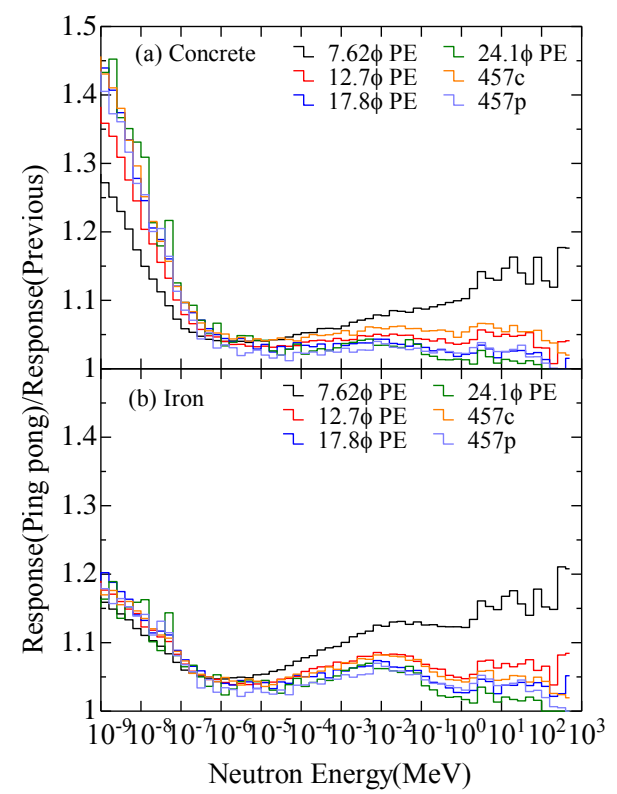

Figure 3. Ratio of the previous response function and the response function including the ping-pong effect for (a) concrete and (b) iron.

evaluated with the MCNPX code. Figure 3 shows the ratio of the previous response function and the response function including the ping-pong effect. Figure 3 indicates the ping-pong effect for the concrete block is more conspicuous than that for the iron block in the low energy region. The response function including the ping-pong effect was used in the data analysis to obtain more precise results. 


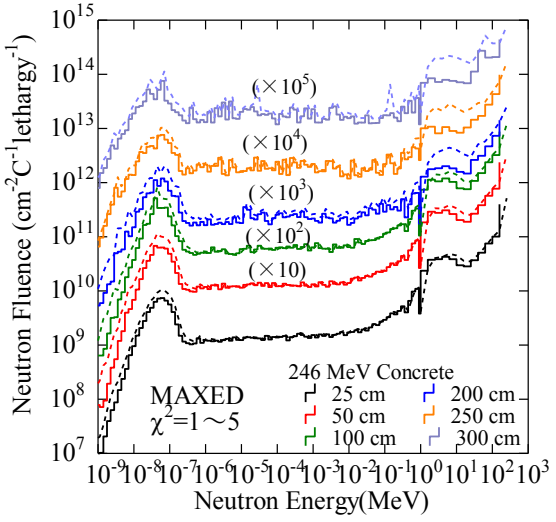

Figure 4. Measured neutron spectra behind the concrete shield using the 243.5-MeV quasi-monoenergetic neutrons. Dotted lines show the simulated spectra.

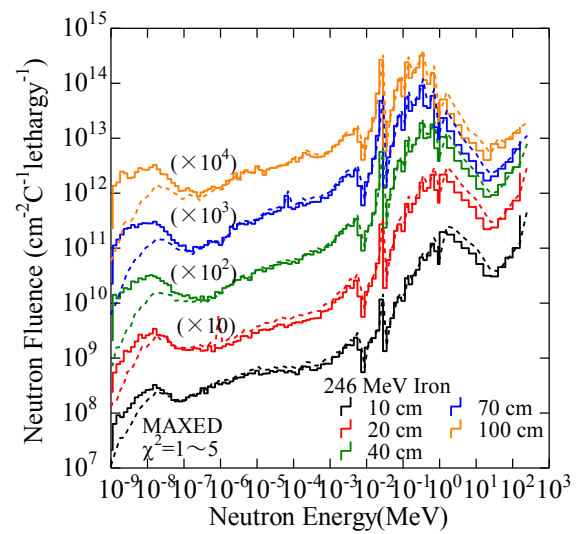

Figure 5. Measured neutron spectra behind the iron shield using the 243.5-MeV quasi-monoenergetic neutrons. Dotted lines show the simulated spectra.

\section{Data analysis}

Neutron energy spectra behind the shields were obtained by unfolding method using the MAXED code [12]. The measured neutron source energy spectra using the TOF method were used to simulate the neutron energy spectra behind the shielding materials in the MCNPX code with rather simple geometrical modeling without a floor and walls of the experimental room. The simulated neutron energy spectra were used for initial spectra in the MAXED code. From the neutron energy spectra with the BSS, the ambient dose equivalent was obtained using conversion coefficients based on the International Commission on Radiological Protection recommendations in 1990 [13, 14].

\section{Results and discussion}

Figures 4, 5, 6 and 7 show the neutron spectra penetrated behind the concrete or iron shields for the quasi-monoenergetic neutrons generated by the $246-\mathrm{MeV}$ and $389-\mathrm{MeV}$ protons. The neutron fluences are converted to the lethargy fluences at $1 \mathrm{~m}$ away from the Li target per proton beam charge (coulombs) in

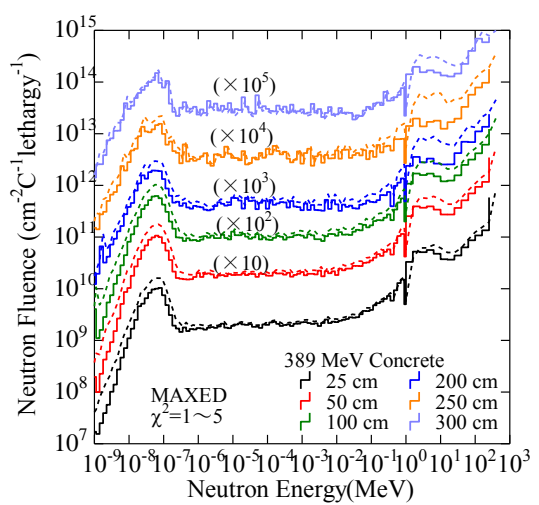

Figure 6. Measured neutron spectra behind the concrete shield using the 386.6-MeV quasi-monoenergetic neutrons. Dotted lines show the simulated spectra.

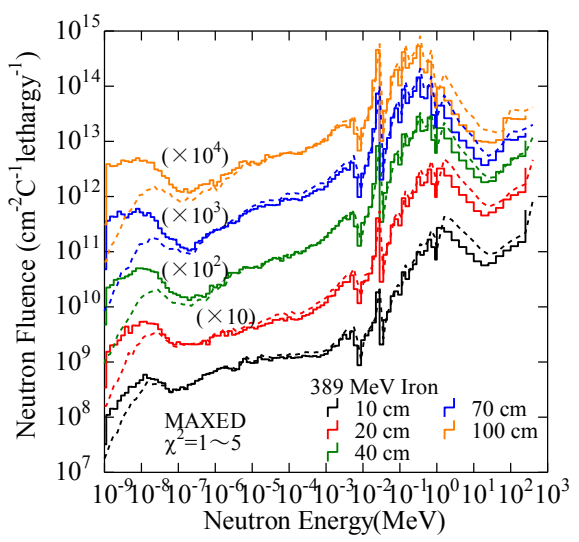

Figure 7. Measured neutron spectra behind the iron shield using the 386.6-MeV quasi-monoenergetic neutrons. Dotted lines show the simulated spectra.

figures 4, 5, 6 and 7. For the unfolding calculations, $\chi^{2}$ static of the solution [12] is obtained from the measured counts and the predicted counts based on the initial spectrum and the response functions for each Bonner sphere. For all spectra, the $\chi^{2}$ static shows values from 1 to 5 , which indicates validity of the unfolded results. These figures indicate that thermal neutrons produced by slowing down in the shielding material are clearly observed. The thermal neutron component also includes room scattered neutrons. It is necessary to correct the contamination of scattered neutrons by calculations, which will be performed in future. Figures 8 and 9 show the preliminary results of neutron ambient dose equivalents per proton beam charge (coulombs) behind the concrete and iron shields as a function of the shield thickness, respectively. The neutron ambient dose equivalents are converted to those at $1 \mathrm{~m}$ away from the Li target in figures 8 and 9. In the shielding experiments, other measurements with a 25.4-cm-diameter and 25.4-cm-thick NE213 scintillator were also performed. The final results of neutron ambient dose equivalents and the attenuation length will be obtained after comparing with the experimental data obtained by the NE213 scintillator in fast energy region and calculated results in all energy region in future work. 


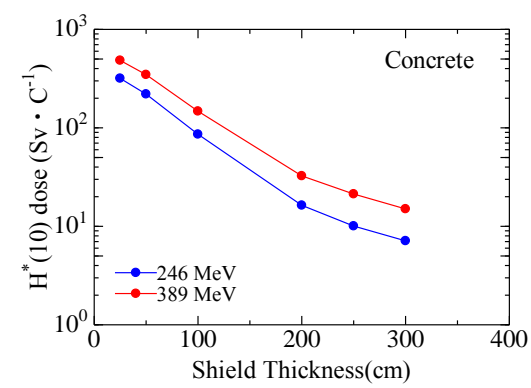

Figure 8. Ambient dose equivalents as a function of the shield thickness behind the concrete shield

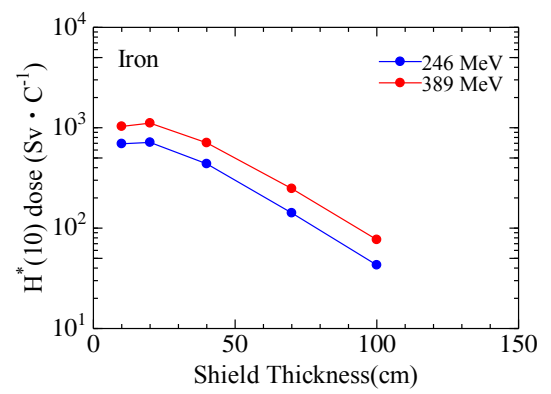

Figure 9. Ambient dose equivalents as a function of the shield thickness behind the iron shield

\section{Conclusions}

The neutron energy spectra were measured through the concrete and iron shields for the quasi-monoenergetic neutrons produced by the ${ }^{7} \mathrm{Li}(\mathrm{p}, \mathrm{xn})$ reaction with the $246-\mathrm{MeV}$ and $389-\mathrm{MeV}$ protons. The neutron energy spectra behind the shielding blocks of concrete and iron were obtained successfully using the BSS, respectively. These experimental data will be useful to perform accurate shielding design and to investigate the accuracy of calculation codes and evaluate neutron cross section data.

\section{Acknowledgements}

The authors wish to thank the accelerator staff of RCNP for helpful co-operation during this experiment.

\section{References}

[1] T. Ishikawa, Y. Miyama and T. Nakamura, Neutron penetration through iron and concrete shields with the use of $22.0-$ and $32.5-\mathrm{MeV}$ quasi-monoenergetic sources, Nucl. Sci. Eng. 116 (1994), pp. 278-290.

[2] N. Nakao, H. Nakashima, T. Nakamura, S. Tanaka, S. Tanak, K. Shin, M. Baba, Y. Sakamoto and Y. Nakane, Transmission through shields of quasi-monoenergetic neutrons generated by 43and $68-\mathrm{MeV}$ protons - I: Concrete shielding experiment and calculation for practical application, Nucl. Sci. Eng. 124 (1996), pp. 228-242.

[3] H. Nakashima, N. Nakao, S. Tanaka, T. Nakamura,
K. Shin, S. Tanaka, H. Takada, S. Meigo, Y. Nakane, Y. Sakamoto and M. Baba, Transmission through shields of quasi-monoenergetic neutrons generated by $43-$ and $68-\mathrm{MeV}$ protons - II: Iron shielding experiment and analysis for investigating calculation methods and cross-section data, Nucl. Sci. Eng. 124 (1996), pp.243-257.

[4] N. Nakao, M. Nakao, H. Nakashima, S. Tanaka, Y. Sakamoto, Y. Nakane, S. Tanaka and T. Nakamura, Measurements and calculations of neutron energy spectra behind polyethylene shields bombarded by 40- and 65-MeV quasi-monoenergetic neutron sources, J. Nucle. Sci. Technol. 34 (4) (1997), pp. 348-359.

[5] H. Yashima, H. Iwase, M. Hagiwara, Y. Kirihara, S. Taniguchi, H. Yamakawa, K. Oishi, Y. Iwamoto, D. Satoh, Y. Nakane, H. Nakashima, T. Itoga, N. Nakao, T. Nakamura, A. Tamii and K. Hatanaka, Benchmark experiment of neutron penetration through iron and concrete shields for hundreds-of-MeV quasi-monoenergetic neutrons I: Measurements of neutron spectrum by a multimoderator spectrometer, Nucl. Technol. 168 (2009), pp. 298-303.

[6] Y. Iwamoto, M. Hagiwara, D. Satoh, H. Iwase, H. Yashima, T. Itoga, T. Sato, Y. Nakane, H. Nakashima, Y. Sakamoto, T. Matsumoto, A. Masuda, J. Nishiyama, A. Tamii, K. Hatanaka, C. Theis, E. Feldbaumer, L. Jaegerhofer, C. Pioch, V. Mares and T. Nakamura, Quasi-monoenergetic neutron energy spectra for 246 and $389 \mathrm{MeV}$ ${ }^{7} \mathrm{Li}(\mathrm{p}, \mathrm{n})$ reactions at angles from 0 to $30, \mathrm{Nucl}$. Instr. Methods A629 (2011), pp. 43-49.

[7] A. Masuda, T. Matsumoto, H. Harano, J. Nishiyama, Y. Iwamoto, M. Hagiwara, D. Satoh, H. Iwase, H. Yashima, T. Nakamura, T. Sato, T. Itoga, Y. Nakane, H. Nakashima, Y. Sakamoto, C. Theis, E. Feldbaumer, L. Jaeferhofer, C. Pioch, V. Mares, A. Tamii and K. Hatanaka, Response measurement of a Bonner sphere spectrometer for high-energy neutrons, IEEE trans. Nucl. Sci. 59 (1) (2012), pp. 161-166.

[8] B. Weigel and A. V. Alevra, NEMUS - the PTB Neutron Multisphere Spectrometer: Bonner sphere and more, Nucl. Inst, Meth. A476 (2002), pp. 36-41

[9] D. B. Pelowitz, MCNPX use's manual Ver 2.5.0, Los Alamos National Laboratory, LA-CP-05-0369 (2005).

[10] Y. Watanabe, K. Kosako, S. Kunieda, S. Chiba, R. Fujimoto, H. Harada, M. Kawai, F. Maekawa, T. Murata, H. Nakashima, K. Niita, N. Shigyo, S. Shimakawa and N. Yamano, Status of JENDL High Energy File, J. Korean Physical Society 59 (2) (2011), pp. 1046-1051.

[11] H. Harano, T. Matsumoto, Y. Tanimura, Y. Shikaze, M. Baba and T. Nakamura, Monoenergetic and quasi-monoenergeti neutron reference fields in Japan, Radiat. Meas. 45 (2010), pp.1076-1082.

[12] M. Reginatto, P. Goldhagen and S. Neumann, 
Spectrum unfolding, sensitivity analysis and propagation of uncertainties with the maximum entropy deconvolution code MAXED, Nucl. Inst. Methods A476 (2002), pp. 242-246.

[13] 1990 Recommendations of the International Commission on Radiological Protection, ICRP Publication 60, International Commission on
Radiological Protection (1991).

[14] T. Sato, A. Endo, M. Zankl, N. Petoussi-Henss, H. Yasuda and K. Niita, Fluence-to-dose conversion coefficients for aircrew dosimetry based on the new ICRP Recommendations, Prog. Nucl. Sci. Technol. 1 (2011), pp. 134-137. 